\title{
Design of Low Noise Amplifier for Optimum Matching between Noise Figure and Power Gain
}

\author{
A. S. Jarallah Al-Jawadi \\ Department of EE \\ Mosul University \\ College of Engineering
}

\author{
A. A. Ismail \\ Department of EE \\ Mosul University \\ College of Engineering
}

\begin{abstract}
The LNA is usually the first stage in any microwave receiver circuit, this stage has an important rule in the quality of the receiver. The design of a LNA in Radio Frequency (RF) circuit requires the trade-off for many importance characteristics such as gain, Noise Figure (NF), stability, power consumption and complexity. This situation Forces designers taking decision in the design of RF circuits. In this paper the aim is to design and simulate a single stage LNA circuit with high gain and low noise using PHEMT transistor at frequency $2.4 \mathrm{GHz}$. The design simulation process is performed using Advance Design System (ADS). A single stage LNA has successfully designed with $16.29 \mathrm{~dB}$ forward gain and $0.44 \mathrm{~dB}$ noise figure and with good stability in frequency of $2.4 \mathrm{GHz}$.
\end{abstract}

\section{Keywords}

Noise figure; gain; LNA; ADS; Stability; S parameters;.

\section{INTRODUCTION}

Low noise amplifier (LNA) is one of the basic building blocks of any communication system. The purpose of the LNA is to amplify the received signal to an acceptable levels while minimizing the noise inside it. The low noise amplifier is used in communication systems to amplify very weak signals captured by the antenna, it is often located very close to the antenna thereby making losses in the feed-line less critical. It is necessary for the LNA to boost the desired signal power while adding very little noise and distortion as possible so that the retrieval of signal is possible in the later stages in the system. Usually Junction Field Effect Transistor (JFETs) and High Electron Mobility Transistor (HEMT) are used in LNA circuits because it's offer high amplification in the first stage, these transistors are energy efficient but reduce the relative amount of shot noise. The general topology of the LNA is consists of three stages: the input matching network, (IMN), the amplifier itself and the output matching network, (OMN). In addition to select the appropriate active component, the IMN and OMN are critical factors in achieving the specified overall amplifier performances. The main topics of the article will include the following steps, bias design, circuit stabilization, noise optimization, input/output matching impedance network and complete circuit characterization. A circuit solution is presented along with complete simulation performance that is required by $2.4 \mathrm{GHz}$ frequency. A number of design tools and iterative operators such as the Smith Chart Utility, Tune Parameter Utility, Optimizer Utility and the Schematic Design are all incorporated in Agilent ADS electronic design automation software, were used to perform the design process [1].

\subsection{LNA Performance Parameters}

The most important performance parameters of a LNA are the power gain and the noise factor $(\mathrm{F})$ or noise figure $(\mathrm{NF})$. The level of the unavoidable noise generated by any two port network is specified by the noise figure which is a comparison of the SNR at the input port of the network to the SNR at the output port as shown in Figure (1) [2]. The noise figure of a receiver system, according to Friiss' formula [3], is given by equation:

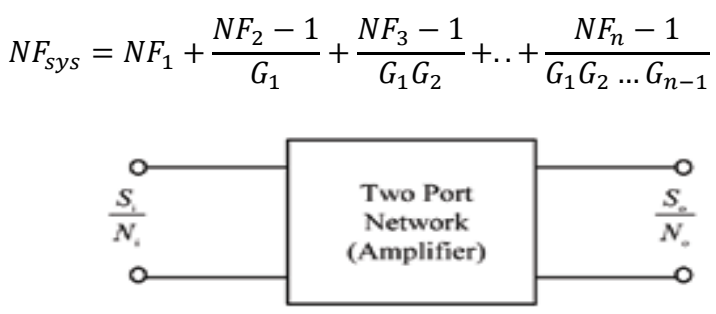

Fig 1: A two port network

Where $\mathrm{Gn}, \mathrm{i}=1,2,3, \ldots$ are the gain parameters in each stages of the receiver. The noise figure (NF) of a system is simply expressed in decibel, that is:

$N F=10 \log 10 F$

Equation (1) shows that the first stage of the receiver will be the most significant contributor to the overall NF of the system. This means that the LNA must be designed for a minimum NF as well as a high gain so that the amplitude of the signal is maximized while adding as minimal amount of noise as possible. The quest for the simultaneous attainment of a high gain and a low NF in the design of a LNA poses a dilemma for the designer, as these are conflicting and mutually exclusive performance requirements. Hence, a tradeoff and subsequent compromise between these two performance parameters is necessary in the design of a single stage LNA [4].

\section{LNA DESIGN AND ANALYSIS}

The design will be done by following the steps given below:

\subsection{Transistor Selection}

This is one of the most important steps in designing a low noise amplifier (LNA). Different types of transistors are available, for example, MESFET, HEMT and PHEMT which can be used for LNA application. According to our specifications, GaAs PHEMT transistor has been used for low noise amplifier due to its low noise figure and high gain. The transistor type ATF-36077 was used because it has high gain at a low noise figure [5]. 


\subsection{Biasing}

In order to design a low noise device, the transistor must be DC biased at an appropriate operating point. These depends on the application used (low noise, high gain, high power), and the type of the transistor (FET, HEMT, etc). The DC biasing requirement for the device is $\mathrm{Vds}$ (Drain to source voltage $)=1.5 \mathrm{~V}, \mathrm{Vgs}($ Gate to source voltage $)=-0.3 \mathrm{~V}$ and Ids(Drain to source current $)=10.57 \mathrm{~mA}$. The biasing technique used for DC biasing of device is active biasing rather than passive biasing because active biasing provides a stable operating point as compared to the passive biasing technique [6]. The I-V curves and the biasing circuit are shown in Figure (2) and Figure (3). The DC biasing circuit must be protected from the high frequency effects of the termination ports so that the S-parameters should not change due to the biasing of the active device. The inductors are inserted between the termination port and DC biasing circuit to protect the biasing circuit from the effects of the termination ports. Another important task is to protect termination ports from the DC voltages and currents used to bias the device, therefore DC blocking capacitors are used [7].

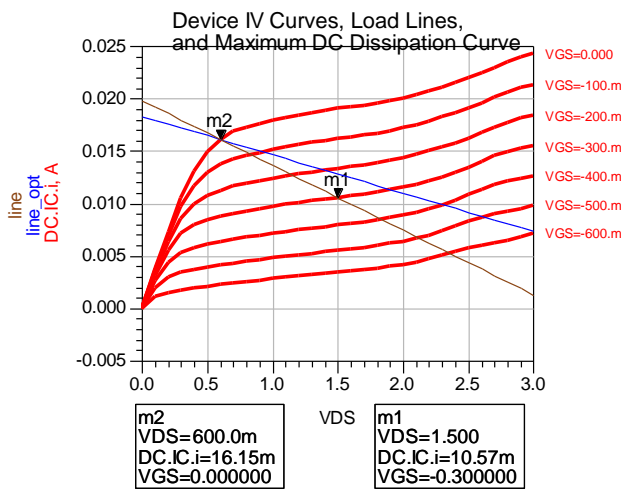

Fig 2: I-V curves for transistor

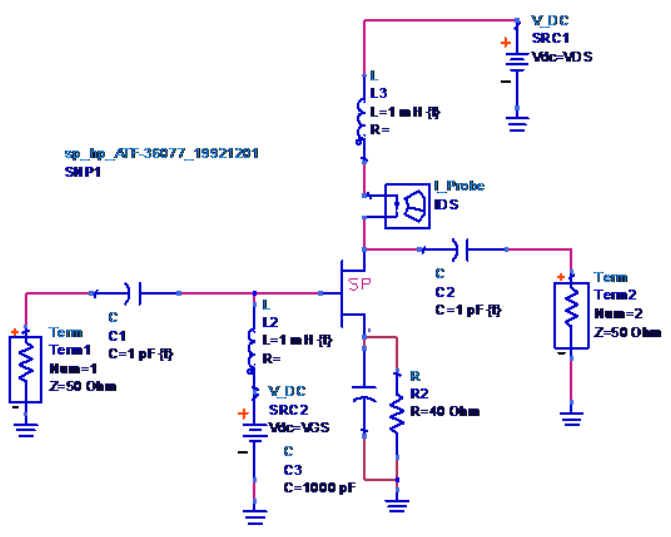

Fig 3: DC biasing circuit

\subsection{Stability Techniques}

Stability analysis should be the next step in LNA design. The S-parameters of the device, the matching networks and the terminations are all used to determine stability. The stability of the amplifier or in other words its resistance to oscillate is a very important consideration in the design. In a two-port network, oscillations are possible when either input or output port presents a negative resistance. This occurs when input or output reflection coefficient is positive $(|\Gamma \mathrm{IN}|>1$ or $|\Gamma \mathrm{OUT}|$
$>1$ ), which for unilateral device occurs when $|\mathrm{S} 11|>1$ or $|\mathrm{S} 22|>1)$. The two port-network shown in figure (4) is considered to be unconditionally stable at operating frequency $2.4 \mathrm{GHz}$ if the real parts of Zin and Zout are greater than (0) for all passive load ZL and source impedance Zs [7].

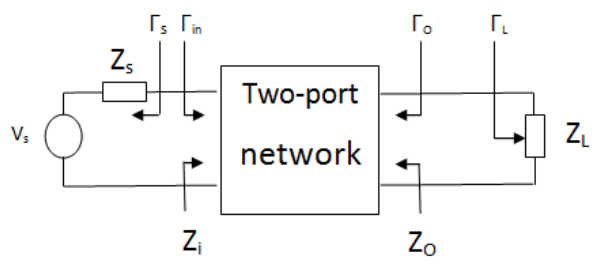

Fig 4: Stability of two-port networks

\subsubsection{Stabilize the Active Device}

The active device in an amplifier must be unconditionally stable for optimum performance of the amplifier. An active device is considered to be unconditionally stable when the Rollet stability factor $(\mathrm{K})>1$ and $|\Delta|<1$. Mathematically, the Rollet stability factor $\mathrm{K}$ and the magnitude of the determinant of the S-parameters $|\Delta|$ are given by equations (3) and (4) as [8]:

$$
K=\frac{1-\left|s_{11}\right|^{2}-|s|_{22}{ }^{2}+|\Delta|^{2}}{2\left|s_{12} s_{21}\right|}>1
$$

$\Delta=S_{11} S_{22}-S_{12} S_{21}<1$

For the selected ATF-36077 PHEMT, K and $|\Delta|$ were calculated to be 0.19 and 0.63 respectively. Clearly, the stability criteria were not met and the active device had to be made unconditionally stable. To put the system in the unconditionally stable region, one can add a series resistor or a shunt conductance to the input or output port. The optimum way to make this active device unconditionally stable and still to provide the lowest possible NF is to load the output port of the active with a suitable resistor [9]. This method should be carefully used because it will reduce the gain of the circuit. A systematic mathematical solution, aided by graphical method using Smith chart, is developed to determine the elements value of circuit stabilization. For high performances of LNA R4,L4 and C4 are optimized to $60.5 \mathrm{ohm}, 0.5 \mathrm{nH}$ and $0.15 \mathrm{pF}$ respectively. The elements stabilization was connected across the output port of the active device as shown in Figure (5) and the resultant simulated stability parameters are shown in Figure (6). Clearly the stability factor $\mathrm{K}$ is greater than unity and the circuit will be unconditionally stable for any combination of source and load impedance.

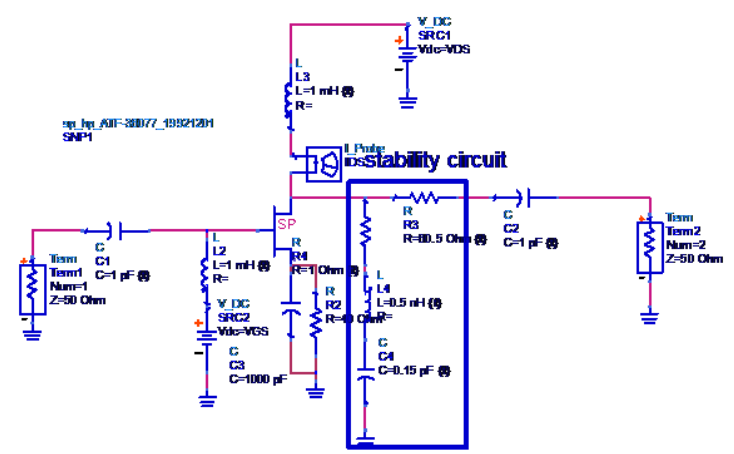

Fig 5: elements stability added to improve stability 


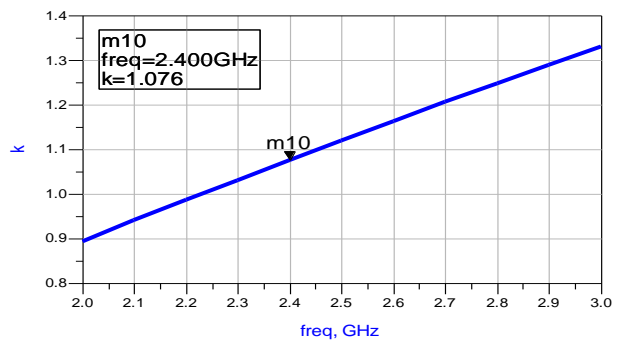

Fig 6: $\mathrm{K}$ factor with circuit stabilization

\subsection{Design Technique}

The single stage low noise amplifier will consist of a transistor and an input and output matching network. Where a matching network is used in both sides of the transistor to transform the input and output impedance $\mathrm{Z} 0$ to the source and load impedance Zs and ZL [10]. The input matching network can serve to match the transistor for optimal input match or for optimum noise, while the second matching network is usually designed to match for optimum gain and output match. To achieve a good input match, the source reflection coefficient must be equal to the conjugate of the input reflection coefficient. However, to obtain optimum noise performance the conjugate of the input reflection coefficient must be equal to Topt Figure (7) shows a typical single stage amplifier design with input and output matching networks $[6,8]$.

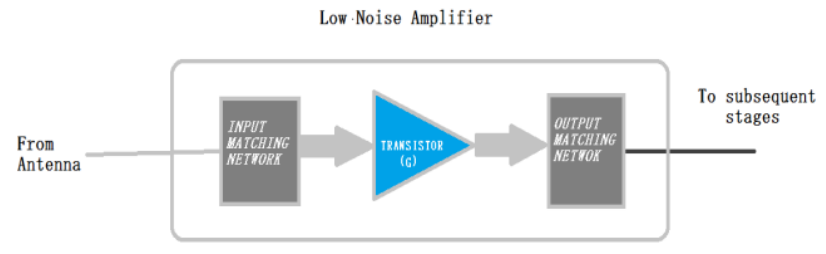

Fig 7 : block diagram of LNA Circuit

\subsubsection{Noise Figure and Available Gain Circles}

The next step in the designing of LNA is to draw constant gain and noise circles on Smith chart as shown in figure (8). ADS providing the constant gain and noise circles on Smith chart tool to find a point on smith chart that gives a high gain and low noise figure because in the design of LNA, the major concern is to minimize the noise and maximize the gain of the input signal. For this purpose some optimum values of $\Gamma \mathrm{s}$ (source reflection coefficient) and $\Gamma \mathrm{L}$ (load reflection coefficient) are selected to design the matching network for which the noise figure is up to a tolerable level and the gain set to the required value. For the matching Network design, the intersection of noise and gain circle has to be located, as shown in figure 8 . From the intersection of the Noise and Gain circles, the value of $\Gamma \mathrm{s}$ is chosen to design the matching network. Then $\Gamma \mathrm{s}$ is used to determine Гout (output reflection coefficient) using the following equation [11]:

$\tilde{\Gamma}_{\text {out }}=S_{22}+\frac{S_{12} S_{21} \tilde{\Gamma}_{s}}{1-S_{11} \hat{\Gamma}_{s}}$
Tout is conjugate matched with $\Gamma \mathrm{L}$. The value of $\Gamma \mathrm{L}$ is used to determine $\Gamma$ in (input reflection coefficient) using the following equation [11]:

$\dot{\Gamma}_{i n}=S_{11}+\frac{S_{12} S_{21} \hat{\Gamma}_{L}}{1-S_{22} \hat{\Gamma}_{L}}$

After the selection of $\Gamma \mathrm{s}$ and $\Gamma L$, the input and output matching networks are designed using ADS Smith Chart utility.

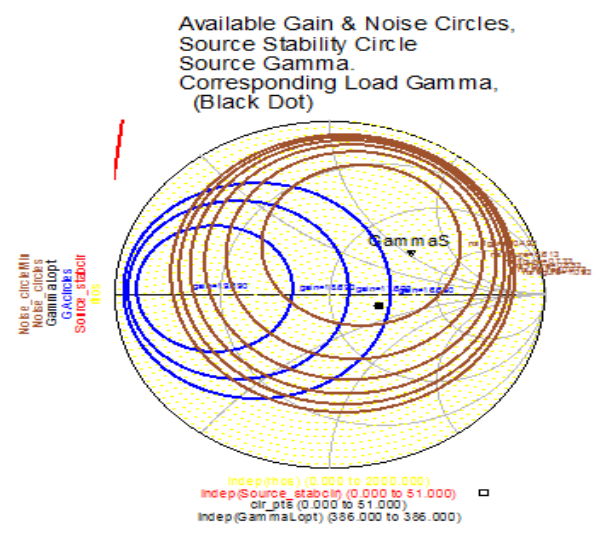

Fig 8: noise figure circles and available gain circles

\subsection{LNA Implementations}

There are two method to implement the previous LNA design, using discrete passive components (lumped element) and using microstrip transmission lines (distributed element models). These two implementations are presented and analyzed below.

\subsubsection{LNA Design With Lumped Element IMN And $O M N$}

The stabilized amplifier was simulated and the results show that the NF is at minimum value and equal to $0.373 \mathrm{~dB}$ when the source impedance Zopt $=52.001+\mathrm{j} 228.937 \Omega$ and the load impedance $\mathrm{ZML}=78.541+\mathrm{j} 101.245 \Omega$. The corresponding power gain is equal to $16.56 \mathrm{~dB}$. This result is shown in Figure (9). The schematic of the LNA including the input and output matching networks implemented with lumped elements is shown in Figure (10).

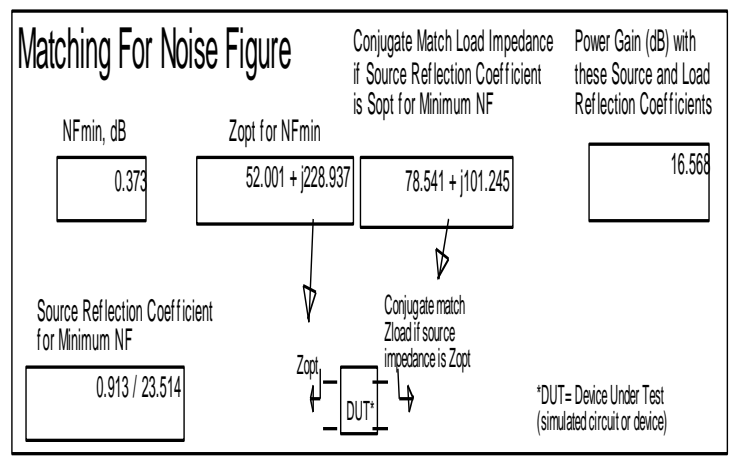

Fig 9 : Matching value for noise figure 


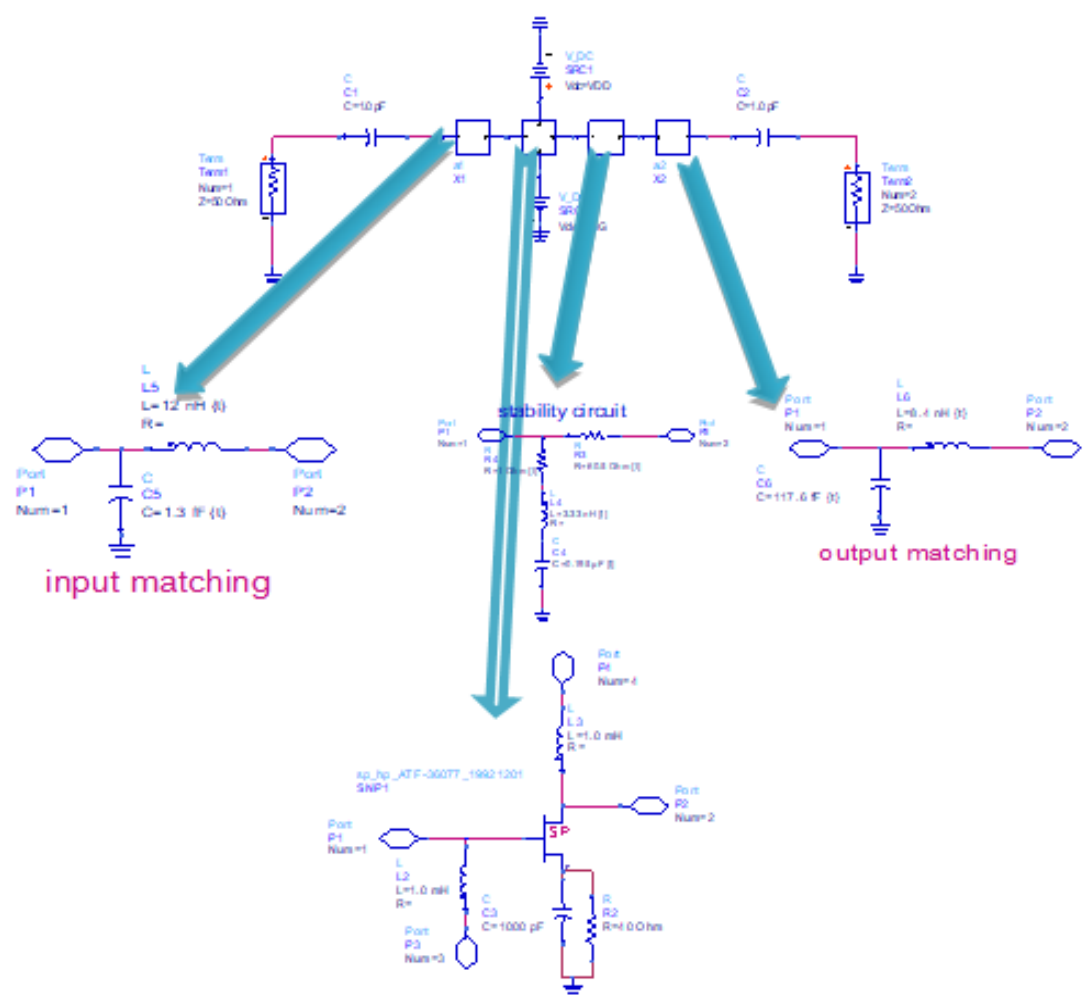

Fig 10 : final Schematic of LNA with lumped element

\subsubsection{LNA Design With Distributed IMN and}

\section{$O M N$}

The second design of the LNA, using microstrip lines and thus distributed models, The lengths and widths for the stub and microstrip line of the matching networks are calculated using the Line Calculator utility in ADS. Once the matching network has been designed and implemented, the single stage LNA can be simulated. The next step is to optimize the results. The optimization tool of the ADS is used for the optimization of matching networks. Housing effects are also included while doing simulations. The schematic of single stage LNA is shown in figure (11).

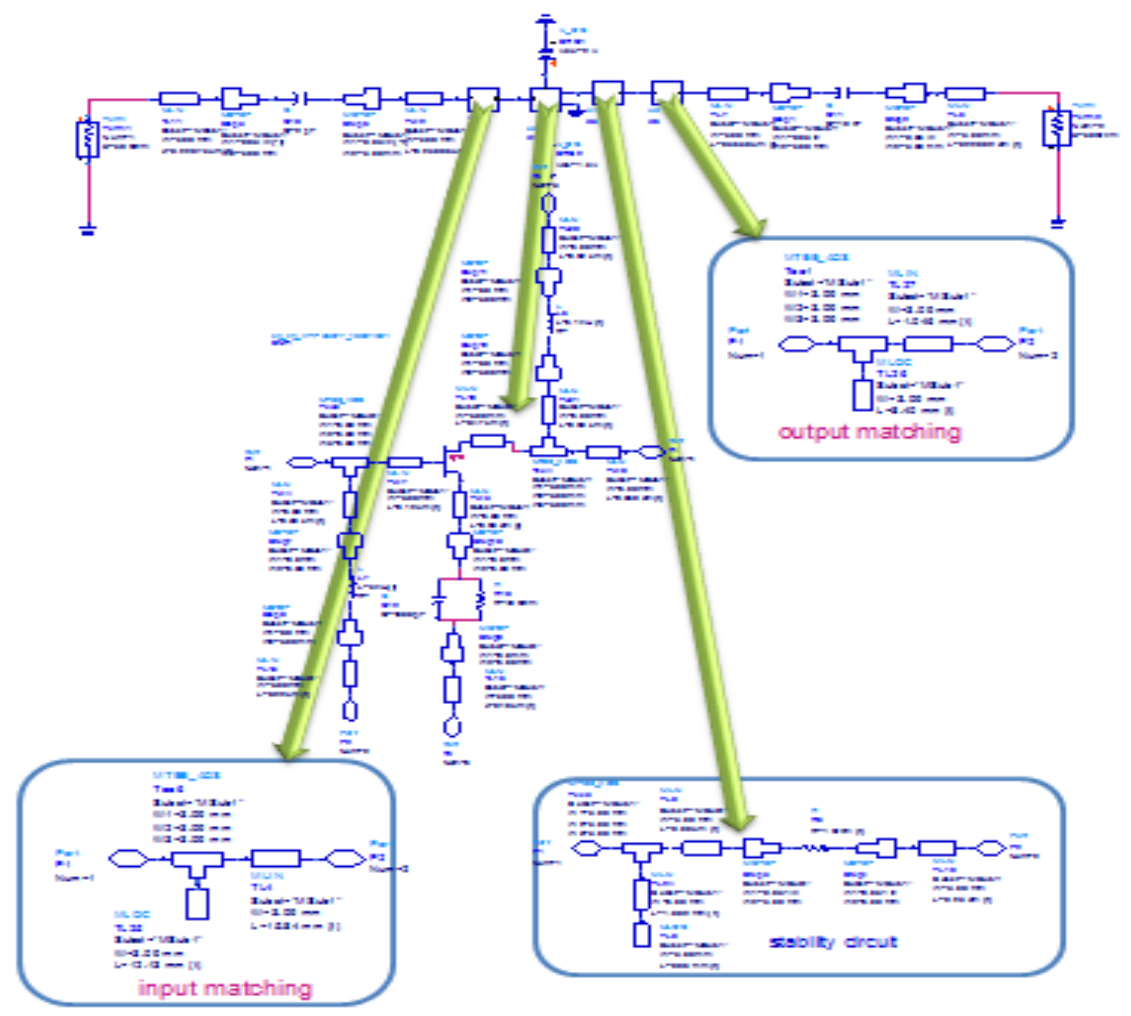

Fig 11: final Schematic of LNA with microstrip line 


\subsection{SIMULATION RESULTS OF LNA}

The Simulation Results of LNA designed are with the followed:

\subsubsection{Simulation Result of LNA with lumped \\ Element}

The (ADS) 2009 is used to optimize the values of inductors and capacitors in order to achieve the input and output impedance matching to obtain a relatively higher gain together with a smaller noise figure, and to keep stability factor greater than one. Figure (12) shows a plot of the power gain (S21) and the noise figure (NF) versus frequency for the
LNA. At a $1.5-\mathrm{V}$ supply, the LNA achieves a forward gain of $17.5 \mathrm{~dB}$ and a NF of $0.44 \mathrm{~dB}$ at $2.4 \mathrm{GHz}$. The power consumption is only $15.7 \mathrm{~mW}$, with a bandwidth equal to 270 MHz. Figure (13) shows the input and the output reflection coefficients (S11 and S22). S11 and S22 are $-7.3 \mathrm{~dB}$ and -22.8 $\mathrm{dB}$, respectively. The input and the output matching circuits at $2.4 \mathrm{GHz}$ are seems to have a good value. The reverse isolation for the LNA is shown in Figure (14) which has an isolation value more than $25.2 \mathrm{~dB}$. Also the stability factor K Plot is shown in Figure (15). Thus, the design LNA should be working stably in frequency range of $2.3 \mathrm{GHz}$ to $3 \mathrm{GHz}$.

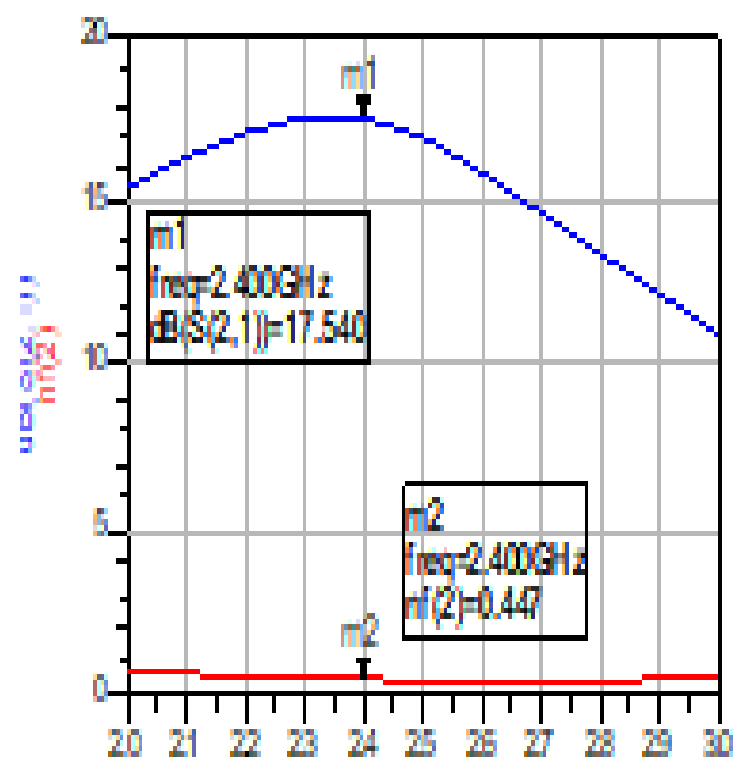

tor ght

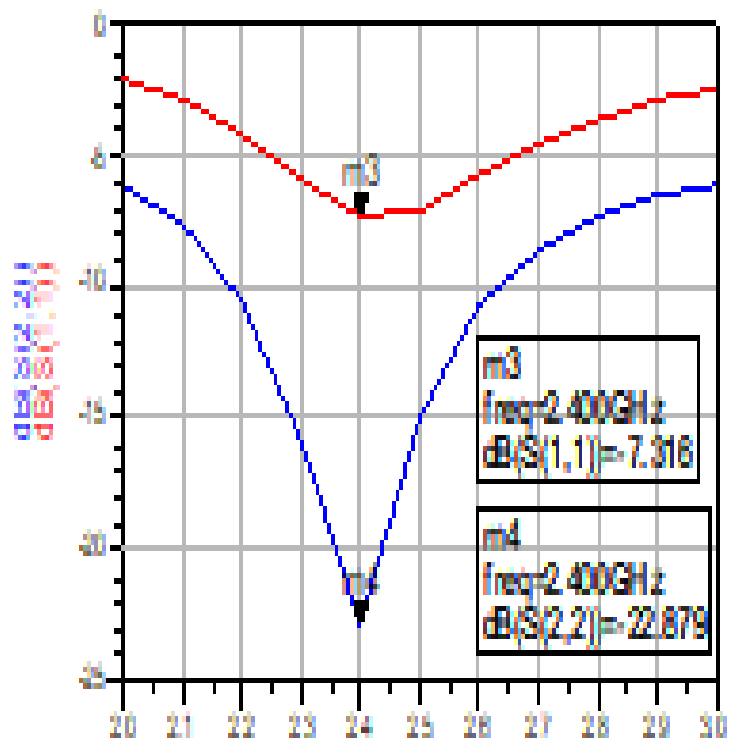

to.

Fig 13:Input and output reflection coefficient

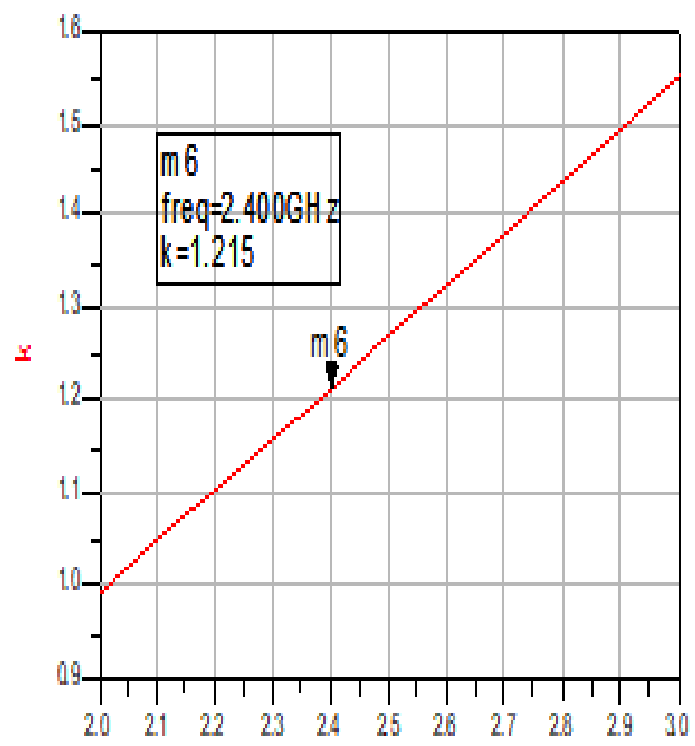

freo. $\mathrm{GHz}$

Fig 14:Reverse isolation(S12) 


\subsubsection{Simulation Result of LNA with Microstrip}

Line

The simulated results include S-parameter, Noise Figure and Stability are shown in figure (16),(17) and (18). The Sparameter simulation results are shown in figure (16). the LNA was simulated at $2.4 \mathrm{GHz}$ and the results show that the
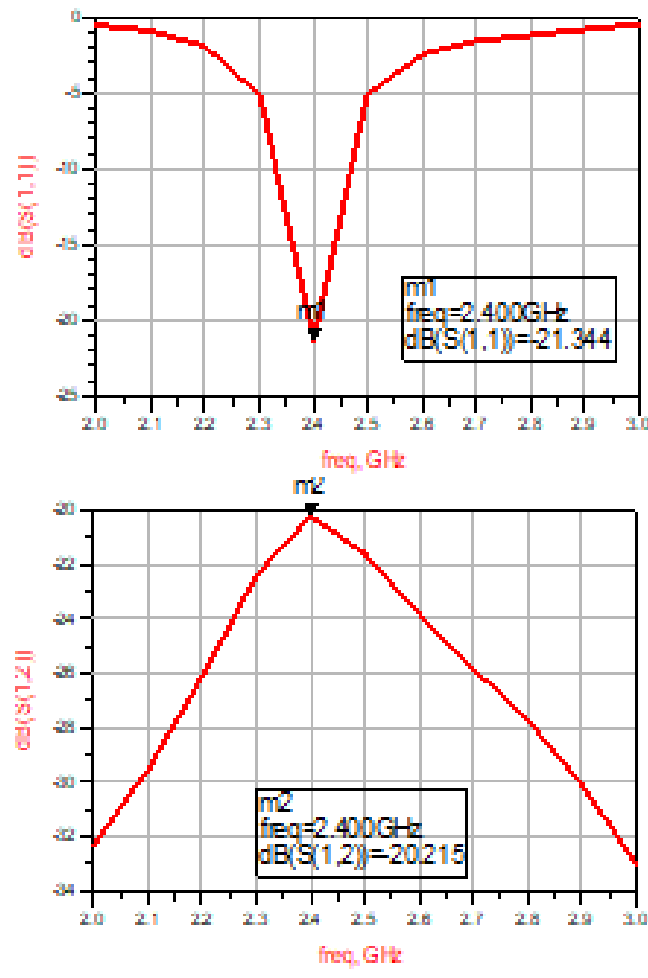

forward gain (S21), insertion loss (S12), input return loss (S11) and output return loss (S22) are $16.29 \mathrm{~dB},-20.215 \mathrm{~dB}$, $21.3 \mathrm{~dB}$ and $-16.1 \mathrm{~dB}$ respectively. From figure (17) the noise figure of $0.382 \mathrm{~dB}$ is obtained at $2.4 \mathrm{GHz}$, Also from figure (18), The stability factor is 1.066 , which is greater than 1 ,thus, the designed LNA is operating with good stability in the frequency range of $2.3 \mathrm{GHz}$ to $3.0 \mathrm{GHz}$.
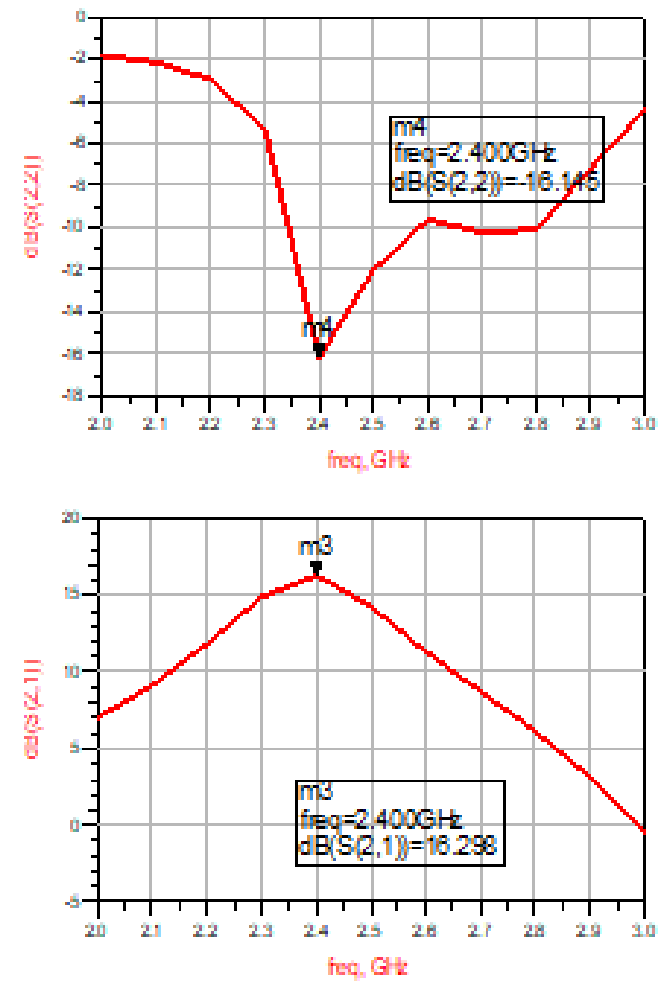

Fig 16 : S-Parameter of LNA with microstrip line

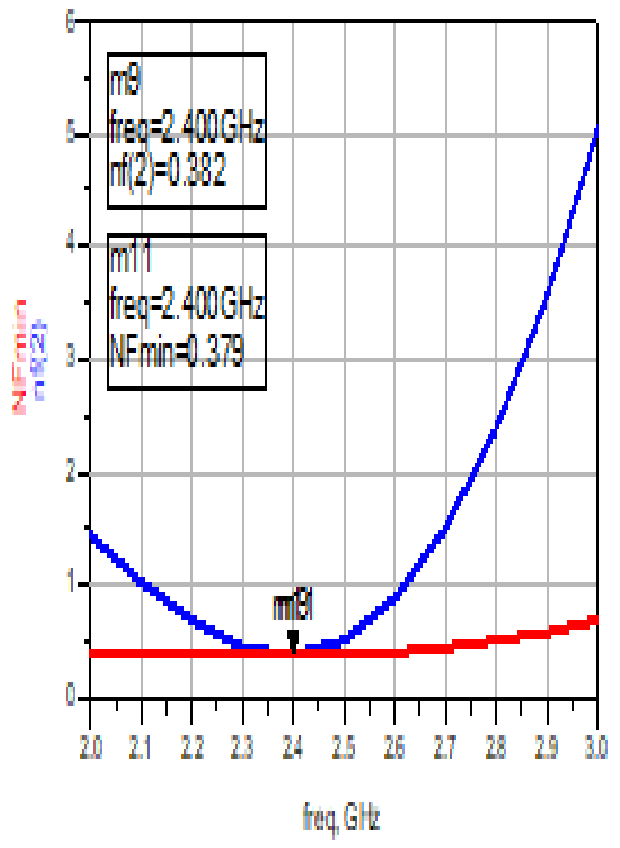

Fig 17 :noise figure of LNA with microstrip line

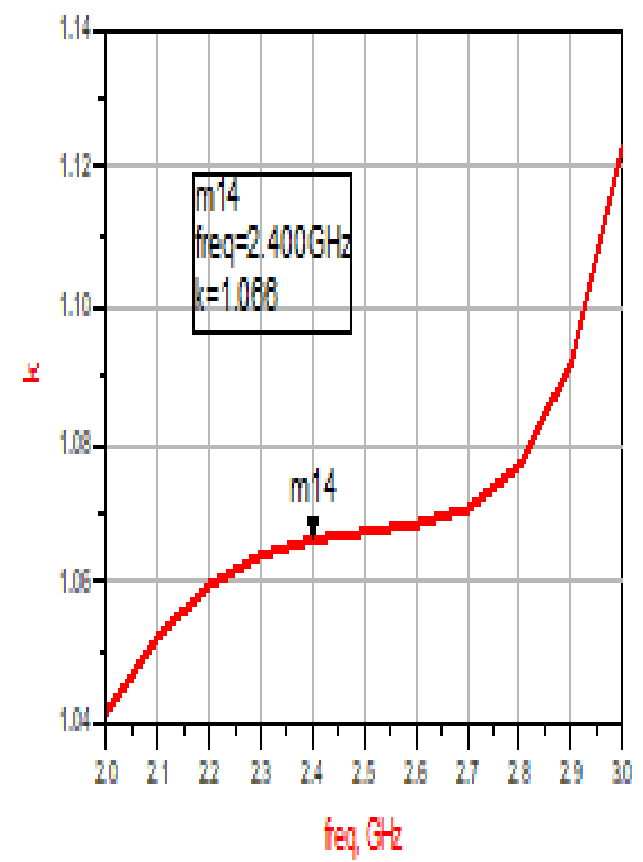

Fig 18 :Stability factor(K) of LNA with microstrip line 


\subsection{Layout, Fabrication and Experimental Results}

The LNA was fabricated using microstrip IMN and OMN. Figure (19A) and (19B) show the ADS layout and the photograph of the LNA test module, respectively. The practical circuit of LNA is shown in Figure (20) realized on a FR-4 substrate $(€ \mathrm{r}=4.3, \mathrm{~h}=1.6 \mathrm{~mm})$. The amplifier has been characterized in small signal condition, at the design bias conditions, resulting in $\mathrm{VDS}=1.5 \mathrm{~V}$ and $\mathrm{VGS}=-0.3 \mathrm{~V}$.

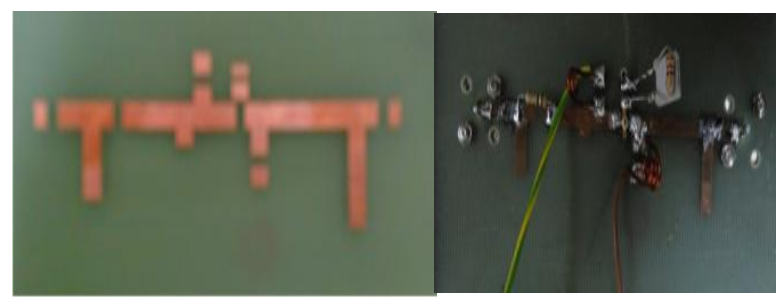

(a) Layout

(b) processed test module

Fig 19 : (a) Layout and (b) processed test module

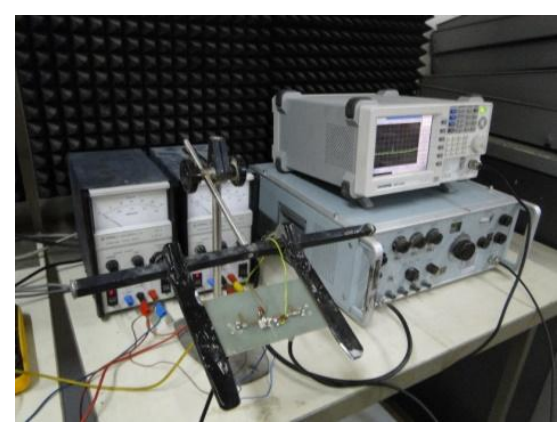

Fig 20 : The fabricated of LNA

The measured output of the amplifier is shown in Figure (21). The practical results show that the output signal is equal to($44.8 \mathrm{dBm}$ ). The power gain of the amplifier is equal $13.5 \mathrm{~dB}$ which very close to result obtained from ADS simulation . The different between the simulated results and practical results can be attributed to the type of circuits used wiring connection which causes a drop voltage across these wiring and connection. Also these wiring can make some stray capacitance which give an error between practical and simulated results.

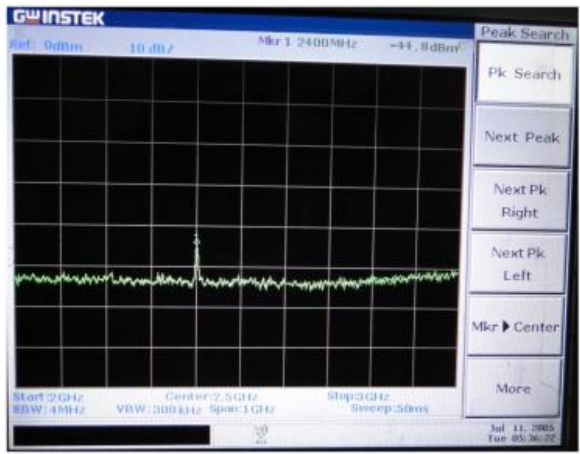

Fig 21 : output signal of fabricated LNA

\section{CONCLUSIONS}

The low noise amplifier (LNA) is a crucial part in RF receiver. It is designed for selecting and amplifying weak signals in certain frequency, reducing noise, and providing an appropriate working condition for the following mixer. The important parameters in a LNA were analyzed firstly such as noise figure, input and output impedance matching network and gain that reflect the quality of the LNA. The LNA was designed at $2.4 \mathrm{GHz}$ operating frequency, the designed LNA has successfully developed with $16.29 \mathrm{~dB}$ gain and $0.382 \mathrm{~dB}$ noise figure.

\section{REFERENCES}

[1] W. Xiao-meil and others, "Design of X-band Low Noise Amplifier for Optimum Matching between Noise and Power", International Conference on Education Technology and Computer(ICETC), IEEE, 2010

[2] T. Thai, " Low Noise Amplifier Design for Operating Frequency of 4.2GHz", ECE 4415a Project, Dec, 2007.

[3] llahnejad, Mohammad, Yasaman Najmabadi, and Alireza Kashaniniya. "Design and Simulation of Low Noise Amplifier at $10 \mathrm{GHz}$ By Using GaAs High Electron Mobility Transistor." IOSR Journal of Electrical and Electronics Engineering 10.5 (2015): 29-34.

[4] Othman, A. R., et al. "Low Noise Figure and High Gain Single Stage Cascoded LNA Amplifier With Optimized Inductive Drain Feedback for WiMAX Application." International Journal of Engineering and Technology (IJET) 5.3 (2013).

[5] Mudavath, Mahesh, and K. Hari Kishore. "Design of RF Front-End CMOS cascade CS Low Noise Amplifier on $65 \mathrm{~nm}$ Technology process." International Journal of Pure and Applied Mathematics 115.7 (2017): 417-422

[6] N. Shoaib, M. Ahmed, I. Mahmood, "Design, Fabrication \& Testing of Low Noise Amplifier at $\mathrm{Ku}$ Band", 2nd International Conference on Advances in Space Technology (ICAST), Proceedings of ICAST. Vol. 2,p.18-23, Pakistan, Nov, 2008.

[7] A. Hamdi, " Design, Analysis and Optimization Technique of 1.9GHz CDMA Low Noise Amplifier", 5th International Conference: Sciences of Electronic, Technologies of Information and Telecommunications (SETIT), Tunisia, March, 2009

[8] Bhale, V. P., and U. D. Dalal. "Design and Optimization of CMOS 0.18 [mu] m Low Noise Amplifier for Wireless Applications." International Journal of Information and Electronics Engineering 4.2 (2014): 92.

[9] Rahimian, Ardavan, and Davood Momeni Pakdehi. "Design and Realization of an S-Band Microwave LowNoise Amplifier for Wireless RF Subsystems." arXiv preprint arXiv:1409.2141 (2014).

[10] Othman, A. R., et al. "High Gain Cascaded Low Noise Amplifier Using T-Matching Network." Journal of Telecommunication, Electronic and Computer Engineering (JTEC) 2.1 (2010): 63-69.

[11] Mohod, Mr Mayur K., and Mr VB Padole. "Design and Analysis of Low Noise Amplifier for $2.47 \mathrm{GHz}$, build for Wireless LAN and WI-FI (802.11 G Protocol)." 